any purposes where great hardness and strength are essential.

Investigation of Gaseous Explosions.Brief reference must also be made-and it will be gratifying to do so-to the important work of one of the committees of the British Association appointed in 1908, under the chairmanship of the late Sir William Preece, for the investigation of gaseous explosions, with special reference to temperature. The investigations of the committee are contained in seven yearly reports up to 1914. Of the very important work of the committee I wish to refer to one investigation in particular, which has proved to be a guiding star to the designers and manufacturers of internal-combustion engines in this country. The members of the committee more directly associated with this particular investigation were Sir Dugald Clerk, Professor Callendar, and the late Professor Bertram Hopkinson.

The investigation showed that the intensity of the heat radiated by the incandescent gases to the walls of the cylinder of a gas-engine increases with the size of the cylinder, the actual rate of this increase being approximately proportional to the square root of the depth of the radiating incandescent gas; the intensity was also shown to increase rapidly with the richness of the gas. It suffices now to say that the heat in a large cylinder with a rich explosive mixture is so intense that the metal eventually cracks. The investigation shows why this occurs, and by doing so has saved enormous sums to the makers of gas- and oil-engines in this country, and has led them to avoid the large cylinder, so common in Germany before the war, in favor of a multiplicity of smaller cylinders.

Charles A. Parsons

( $T$ o be continued)

\section{A QUESTION CONCERNING THE NATURE OF VELOCITY}

Tolman's remarkable success in deriving by means of his principle of similitude ${ }^{1}$ a large number of physical laws, laws which have also been otherwise derived by the more natural and usual method of experimentation and measurement, ought to indicate that there is probably something fundamentally right about his method of procedure. His argument involves two universes, while physics knows only one-and the bearing of his conclusions upon the single universe that we know is not altogether apparent. When he further asks it to be assumed that the velocity of light and the charge of the electron shall be the same in both universes, his argument seems far removed from the facts of the laboratory and its relevance to the usual physics may be, and indeed has been, brought into serious question.

But his argument may be developed without any appeal to two universes. So developed it has an important bearing upon the theories of the nature of electricity and of the manner of the propagation of light. Consider any two observers in our present universe, each of whom with a different set of standards of measurement makes experiments and determines laws. The laws determined by the two observers will have the same algebraic form and will differ only in the value of the constants which they involve. Since all of the measureable quantities of physics are defined in terms of three fundamental and ultimately undefined quantities, each observer will need only three standards ${ }^{2}$ in order that he may

1 Tolman, Phys. Rev., 3, 244, 1914; 4, 145, 1914; $6,219,1915 ; 8,8,1916 ; 9,237$, 1917. Buckingham, ibid., 4, 345, 1914. Nordstrom, Finska Vetenskaps Soc. Forh., 57, 1914-15; Afd. A. No. 22. Ishiwara, Science Report of Tohoku Imp. Univ., 5, 33, 1916. Ehrenfest-Afanassjewa, Phys. Rev., 8, 1, 1916. Bridgman, ibid., 8, 423, 1916. Karrer, ibid., 9, 290, 1917.

2 All seem agreed that length and time are fundamental and undefinable. About the third quantity, force or mass, or energy, as the case may be, there seems to be considerable debate. The argument of the present paper is valid, whichever one of them is favored. I have chosen force be- 
measure all quantities which come within his observation. The standards of the two observers, $O$ and $O^{\prime}$, may be connected by algebraic equations, for length, $l^{\prime}=x l$, for time $t^{\prime}=y t$, and for force, $f^{\prime}=z f$, where $x, y$ and $z$ are constants which may have any value and which express the ratio between the magnitudes of the corresponding standards of the two observers. From these three equations corresponding "transformation equations," involving $x, y$ and $z$, for all of the measureable quantities of physics may be calculated. Suppose now that the two observers got together and $O$ says to $O^{\prime}$ :

Surely it is the privilege of each of us to work with whatever standards he prefers, you prefer foot-seconds while I prefer gram-centimeters; yet let us, for such and such reasons and for our mutual convenience, each still keeping his standards different from those of the other, so alter our standards that we can both report all electrical charges and all velocities by precisely the same numerical value.

\section{$O^{\prime}$ may be conceived to reply:}

We know indeed that electrical charges are made up of a number of ultimate charges or electrons and that each of these little charges has the same magnitude. If we report all charges by the same number, that is virtually the same as each of us reporting the same count. I see that in favor of

cause it seems to me to be primary in the course of experience, not because it makes any difference in the general argument. Many physicists, perhaps most, are disposed to believe that there are two additional fundamental and ultimately undefined quantities necessary to complete the structure of modern physics, namely, (1) temperature or entropy (for thermodynamics) and (2) quantity of electricity or magnetic permeability (for electromagnetics). But temperature is defined by the gas-law, and charge is defined by Coulomb's law. These laws, discovered by the experimentation of workers in whose minds temperatures and charges (respectively) were regarded as equal if in similar situations they produced equal effects, have gradually come in the history of science to assume the sacred functions reserved for definitions. It can not be asserted too strongly that they are not "laws." This, however, is methodology, not theoretical physics, and not the central argument of the present paper. your suggestion, but I know of no corresponding consideration in regard to velocity. I see that there is only one really correct way of reporting the magnitude of charges. But I do not see that such is the ease for velocities.

Suppose however, that $O$ and $O^{\prime}$ do put the agreement into effect. Then $v^{\prime}=v$, and $e^{\prime}=e$.

Then

Also

$$
v^{\prime}=\frac{v^{\prime}}{b^{\prime}}=\frac{x l}{y t}=\frac{x}{y}(v)=v ; \text { and } x=y .
$$

$e^{\prime}=\sqrt{f^{\prime} l^{2}}=\sqrt{2 f x^{2} l^{2}}=x \sqrt{z} \cdot \sqrt{f l^{2}}=x \sqrt{z} \cdot e=e ; \quad$ and $\quad z=\frac{1}{x^{2}}$.

Transformation equations involving only one unknown quantity, $x$, may now be written connecting all of the measurable quantities of $O$ with the corresponding quantities of $O^{\prime}$. These equations are identical with those of Tolman; and $O$ and $O^{\prime}$, working with the perfectly legitimate ${ }^{3}$ mathematical reasoning that Tolman uses, may derive, by virtue of their simple agreement, all of the conclusions which he derives from his principle of similitude.

So long as $O$ and $O^{\prime}$ have no agreement between themselves, the transformation equations connecting their various quantities will involve three unknown quantities, $x y$ and $z$.

3 To illustrate the method of reasoning-The transformation equation for energy density is

$$
u^{\prime}=\frac{1}{x^{4}} u,
$$

and that for temperature,

$$
T^{\prime}=\frac{1}{x} T .
$$

If energy density is a function solely of temperature, then $u=F(T)$ and $u^{\prime}=F\left(T^{\prime}\right)$ where $F$ in both equations has precisely the same value, for both observers obviously will find the same law. Substituting in the equation $u^{\prime}=F\left(T^{\prime}\right)$, we have

$$
\frac{1}{x^{4}} u=F\left(\frac{1}{x} T\right) \text {. }
$$

But $u=F(T)$, hence

$$
\frac{1}{x^{4}} F(T)=F\left(\frac{1}{x} T\right) .
$$

The only solution of this functional equation is $F(a)=k a^{4}$. Hence $u=k T^{4}$, and Stefan's law has been demonstrated without experiment. 
Only when the number of these unknown quantities is reduced to one, does it become possible for them to make use of the mathematical reasoning, involving the solution of functional equations, which has led in the hands of Tolman to such a wide variety of useful results. Since $x$ is the ratio between their standards of length-measurement, and $y$ that between their standards of time-measurement, they can express $y$ in terms of $x$ if they agree to make the same numerical report about any one kind of quantity which involves both length and time for its definition, that is, if they agree to make the same reports either about velocities or about accelerations. By making a corresponding second agreement about some quantity which involves for its definition force and time or length or both, such for instance as charge or mass or energy, they will be able to express $z$ in terms of $x$ and to derive an entire set of transformation equations which involve only one unknown quantity. With this new set of equations at hand, they may undertake to set up functional expressions and to derive laws as Tolman has done.

Evidently the number of the different possible sets of transformation equations is quite considerable, for there are many measurable quantities in physics which involve for their definition more than one of the three fundamental undefined quantities. I have calculated nine such different sets. Several of them lead to some of the conclusions which may be deduced from the equations of $O$ and $O^{\prime}$ above (based upon agreements concerning velocities and charges); several of them lead, in cases where the set of $O$ and $O^{\prime}$ has proved fertile, to insoluble or absurd functional equations which point to no solution. Some of them lead to laws which are contrary to those whose validity has been established by experiment. None of the sets is as fertile or leads to as many well established laws as the set which is based upon the agreement of $O$ and $O^{\prime}$ to report all charges and all velocities by the same numerical value. But this agreement is the only thing in the way of an assumption which is involved in the simpli- fied form of Tolman's principle of similitude that is developed by $O$ and $O^{\prime}$ of this paper. The noteworthy success of Tolman in deriving from his principle a large number of experimentally valid laws is evidence that an agreement between observers working with different standards of measurement to report the same charges and velocities by the same number is somehow more intimately in harmony with the order of nature than any other similar agreement relative to some other of the quantities of physics.

Electrical charges may be regarded as if they are made up of a countable number of small units. This has been adequately demonstrated by the researches of Millikan and others in which electrons have actually been isolated and counted. But it could also have been predicted-for, as an assumption, it leads in the hands of $O$ and $O^{\prime}$ to many conclusions which are otherwise verified by experimental fact. In the same way the assumption that velocities are of such sort that there is only one right way to report their magnitude, is one which leads, vastly better than any similar assumption, to the deduction of laws which are established in fact. Hence the assumption is probably true.

Professor Tolman has kindly read the first draft of this paper. He suggests that the conclusion that velocity is of such nature that there is only one right way to report its magnitude, a conclusion which has here been reached by abstract reasoning, may be interpreted concretely to mean that "any given velocity is most sensibly regarded as a given fraction of the maximum possible velocity, namely that of light."

Tenney L. Davis

\section{Department of Chemistry, \\ MASSACHUSETTS INSTITUTE OF TECHNOLOGX, CAMBRIDGE, Mass.}

\section{JACQUES DANNE}

WITH the outbreak of the world conflict in 1914 Le Radium at once ceased publication, all of its editors being called into service. The decision for service was no less definite than the assurance that publication would be 